\title{
The Establishment and Application of TRACE/CFD Model for Maanshan PWR Nuclear Power Plant
}

\author{
Yu-Ting $\mathrm{Ku}^{1, \mathrm{a}}$, Yung-Shin Tseng ${ }^{1, \mathrm{~b}}$, Jung-Hua Yang ${ }^{1, \mathrm{c}}$ \\ Shao-Wen Chen ${ }^{2, d}$, Jong-Rong Wang ${ }^{2,3, e}$, and Chunkuan Shih ${ }^{2,3, f}$ \\ ${ }^{1}$ Department of Engineering and System Science, National Tsing Hua University, \\ No. 101, Section 2, Kuang-Fu Rd., Hsinchu, Taiwan \\ ${ }^{2}$ Institute of Nuclear Engineering and Science, National Tsing Hua University, \\ No. 101, Section 2, Kuang-Fu Rd., Hsinchu, Taiwan \\ ${ }^{3}$ Nuclear and New Energy Education and Research Foundation, \\ No. 101, Section 2, Kuang-Fu Rd., Hsinchu, Taiwan \\ a s100011801@m100.nthu.edu.tw, ${ }^{b}$ yungshintseng@gmail.com, ${ }^{c}$ junghua1984@gmail.com, ${ }^{d}$ \\ chensw@mx.nthu.edu.tw, ${ }^{\mathrm{e}}$ jongrongwang@gmail.com, ${ }^{\dagger}$ ckshih@ess.nthu.edu.tw
}

Keywords: CFD; PWR; FLUENT; TRACE

\begin{abstract}
In this study, a three-dimensional model was developed by using CFD and TRACE to simulate the flow behaviors and thermal-hydraulic phenomena in the downcomer and lower-plenum of Maanshan PWR nuclear power plant (NPP) reactor pressure vessel (RPV). Detailed geometries of vessel components such as neutron shield panels, core support, lower core plate, butt-type columns were considered in this model for flow calculations. Important settings of the present CFD model were first validated with the data of ROCOM facility. Following those settings and considering the geometries and operating conditions of Maanshan NPP, the CFD model was built. In order to provide sufficient transient boundary conditions for CFD simulations, TRACE model was coupled with the CFD model. In this study, main steam line break accident (MSLB) was chosen as a transient event for simulations and analyses. It was found that unexpected temperature and pressure variations can be found in vessel within a short period. In addition, the locations of loop inlets and the neutron shielding plate in downcomer may affect the core flow distributions and enhance core cooling rate in some parts of vessel.
\end{abstract}

\section{Introduction}

Maanshan NPP is the third NPP and the only PWR in Taiwan. The rated core thermal power is 2775 MW. The reactor coolant system has three loops, each of which includes a reactor coolant pump and a steam generator. The pressurizer is connected to the hot-leg piping in loop 2.The object of this study is to develop the TRACE/CFD model of Maanshan NPP. And this model was used to perform MSLB transient analysis.

The advanced thermal hydraulic code named TRACE has been developed by U.S. NRC for NPP safety analysis. According to the TRACE manual [1], one of the features of TRACE is its capacity to model the reactor vessel with 3-D geometry. It could support a more accurate and detailed safety analysis of nuclear power plants. TRACE has the greater simulation capability than other legacy codes (TRAC-P, TRAC-B, RELAP5 and RAMONA), especially for events such as LOCA. In our previous research [2], we established Maanshan NPP TRACE model successfully. In PWR, the heat transfer effect and flow mixing phenomenon have significant effects on MSLB. In order to study these flow behaviors, this paper established a RPV model by using Communication CFD code FLUENT [3]. The thermal hydraulic data obtained from FLUENT calculation could be feedback to other code, especially as system code with one-dimensional or simplification three-dimensional vessel module calculation, to improve the accuracy of thermal hydraulic simulation. In addition, the results of TRACE for MSLB transient were used to be as the boundary conditions of CFD model. 


\section{Model description}

During the development of Maanshan NPP CFD model, doing the verifications of meshing scheme, numerical method and turbulence model is necessary. ROCOM is the proper case for these verifications because the geometry of ROCOM experiment facility is similar to Maanshan RPV. In order to find the appropriate meshing scheme, numerical method and turbulence model, we established the ROCOM CFD model by using FLUENT and compared the results of CFD with ROCOM experimental data. These verifications were shown in our previous research [4]. According to the results of the comparison [4], standard k- $\omega$ turbulence model and numerical method setting which were used in ROCOM model were applied in Maanshan CFD model.

Maanshan CFD Model

A three-dimensional Maanshan RPV model, including only downcomer and lower plenum, was built by using CFD code FLUENT (see Fig. 1). The RPV model consists of cold legs, hot legs, neutron shield panels, core support, lower core plate, butt type columns, energy absorber assembly, upper tie-plate, lower tie-plate and secondly core support. Some assumptions and simplifications were employed to develop the geometrical model in this research:

- Upper plenum and core were ignored because FLUENT cannot do the neutron kinetic calculation. For the same reason, model outlet was changed from hot legs to lower core plate.

- In order to compensate for the decrease of flow resistance, because of the simplification above, a porous zone was added at outlet.

- The wall in this CFD model was considered as adiabatic for prevent heat loss while we calculate the flow mixing.

Moreover, an unstructured mesh system was employed to generate meshes in this model. The mesh was roughly about 9.52 million cells with tetrahedron/hexahedral/wedge meshes, and the maximum mesh skewness was controlled below 0.93 . The computer with Intel core i5, 16GB RAM and Windows 7 operating system was used in this paper. This model was assumed as an incompressible, no heat source, and transient problem, the governing equations could be written as: Continuity Equation:

Momentum Equation:

$$
\frac{\partial \rho}{\partial t}+\nabla \cdot(\rho \vec{U})=n k
$$

Energy Equation:

$$
\frac{\partial(\rho \dot{U})}{\partial t}+\nabla \bullet(\rho \vec{U} \vec{U})=-\nabla P+\rho \vec{g}+\nabla \bullet(\mu \nabla \vec{U})
$$

$$
\frac{\partial}{\partial t}(\rho E)+\nabla \cdot(\stackrel{r}{v}(\rho E+p))=-\nabla \cdot\left(\sum_{j} h_{j} J_{j}\right)+S_{h}
$$

SIMPLE-C (Semi-Implicit Method for Pressure-Linked Equations-Consistent) was employed for the pressure-velocity coupling in this case [5,6]. Momentum, volume fraction, turbulent kinetic energy, and turbulent dissipation rate of liquid phases were calculated by using the second order upwind scheme. The convergent residues of energy equation and momentum equation are less than $10^{-5}$, and others are $10^{-3}$. In Maanshan CFD model, the flow path was described as follows: a) Working flow was injected into downcomer via three cold-leg loops; b) The flow field of downcomer was slightly affected by neutron shield panels at inner-wall; c) In the lower-plenum, flow direction was changed due to the geometry of lower-plenum; d) Flow field was affected by complex structure and made non-uniform distribution in lower-plenum; e) Working flow arrived lower core plate, the outlet of CFD model. Boundary conditions of steady-state are based on NPP operating data and listed at Table 1. 


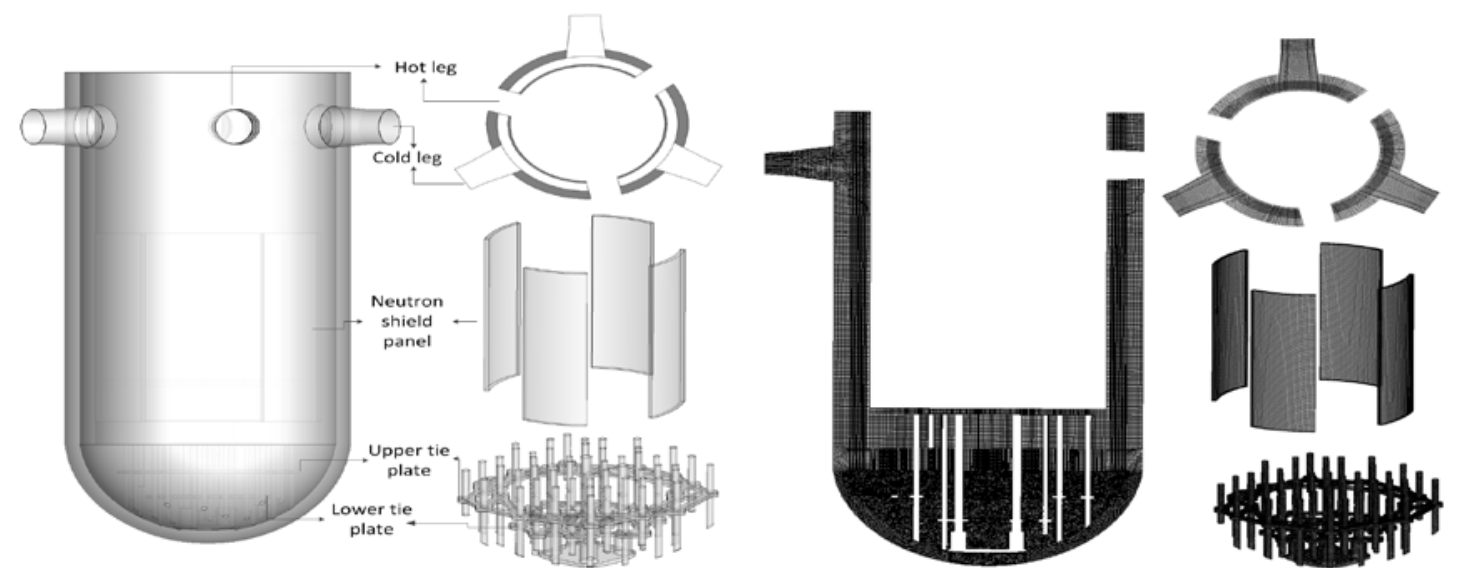

Fig. 1. Maanshan simulation model and mesh distribution

\section{Maanshan TRACE Model}

TRACE is a system code for the NPP safety analysis. Maanshan NPP TRACE model contains 69 hydraulic components, 380 control blocks, 34 heat structures and 2 power components (see Fig. 2). Main components including one 3-D vessel, three reactor coolant system (RCS) loops, one pressurizer, three steam generators and basic plant control systems such as 3-element feedwater control, pressurizer spray, pressurizer level and heater control, and steam dump control. The 3-D vessel component contains 2 radial rings, 6 azimuthal sectors and 12 axial levels. The outer radial ring represents downcomer region and the reactor core is placed in the inner radial ring from axial level 3 to axial level 6. Six control rod guide tubes are connected above the core region. Nuclear fuels are modeled by 6 heat structures (one for each azimuthal sector). Each RCS loop contains hot leg piping, steam generator U-tube, crossover piping, reactor coolant pump, cold leg piping, accumulator tank and accumulator check valve. Pressurizer and pressurizer surge line are connected on RCS loop number 2. In addition, the Maanshan TRACE model had been verified by Maanshan NPP startup test report [2]. Table 2 shows the boundary conditions of major parameters in Maanshan TRACE model.

The steady-state and transient-state calculations were performed in this paper. In MSLB transient, high pressure coolant injection (HPI) and low pressure coolant injection (LPI) were assumed to be failure, and only accumulator (ACC) injection was available. The result of MSLB transient was provided by using Maanshan TRACE model in our previous research [4]. In this model, the break area was $200 \%$ of main steam line cross-section area (double ended guillotine, DEG) and located at loop 3. The major sequence of MSLB transient was shown in Table 3. According to the results of TRACE [4], power tripped when low pressure scram signal was obtained at 1000.05s. The curves of pressure and temperature of three loops show the similar trends. As the pressure reached $4240 \mathrm{kPa}$, ACC injection was initiated to spray coolant water (at 1078s) via loop 2, and thus a higher mass flow rate in loop 2 occurs after ACC injection. The results of TRACE model from 1000.01s to 1100s was utilized as the transient input-data of CFD model.

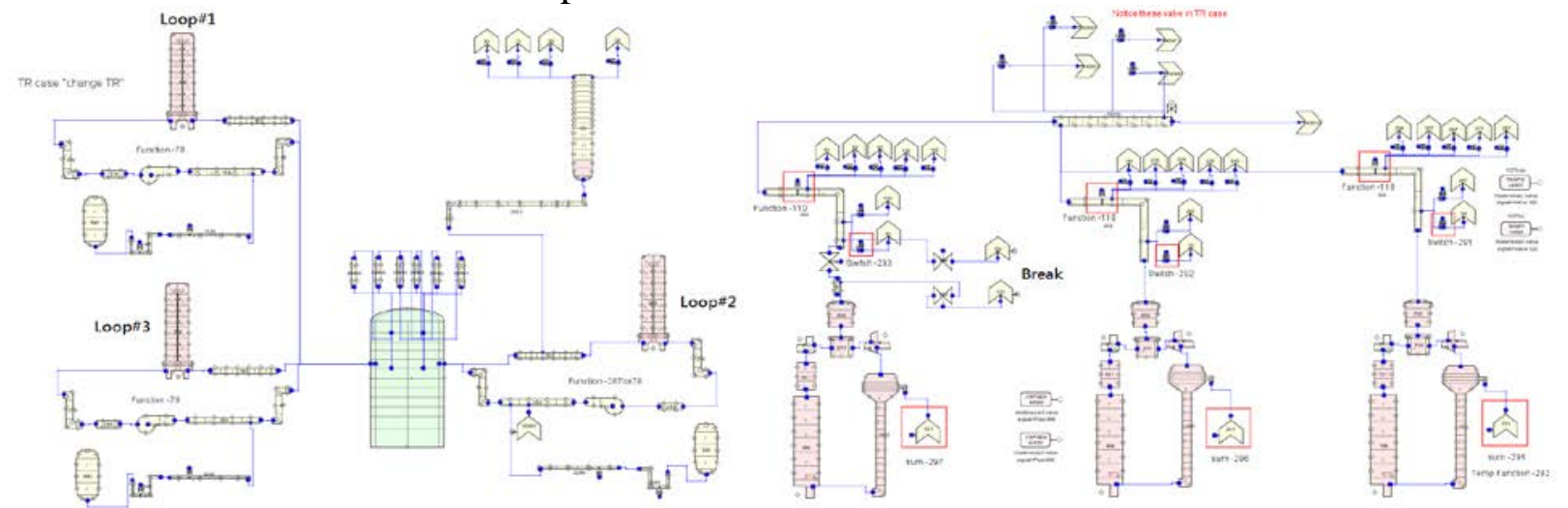

Fig. 2. The TRACE model for Maanshan nuclear power plant with MSLB 
Table 1 Boundary conditions of Maanshan CFD model in normal operation

\begin{tabular}{lc}
\hline \multicolumn{1}{c}{ Boundary conditions } & Values \\
\hline \hline Working Flow Density $\left(\mathrm{kg} / \mathrm{m}^{3}\right)$ & 740 \\
Cold-leq & \\
Mass flow rate $(\mathrm{kg} / \mathrm{s})$ & 4410.18 \\
Temperature $(\mathrm{K})$ & 564 \\
\hline \hline
\end{tabular}

Table 2 Major parameters boundary conditions of Maanshan TRACE model

\begin{tabular}{|c|c|}
\hline Parameter & Values \\
\hline Design pressure (MPa) & 15.6 \\
\hline Maximum core power (MW) & 2775 \\
\hline Primary system volume $\left(\mathrm{m}^{3}\right)$ & $2.15 \times 10^{2}$ \\
\hline Number of loops & 3 \\
\hline \multicolumn{2}{|l|}{ Cold-leq } \\
\hline Inner diameter $\mathrm{D}(\mathrm{m})$ & $7.87 \times 10^{-1}$ \\
\hline Length L (m) & 15.7 \\
\hline $\mathrm{L} / \sqrt{\mathbf{D}}\left(\mathrm{m}^{0.5}\right)$ & 17.69 \\
\hline \multicolumn{2}{|l|}{ Downcomer } \\
\hline Flow area $\left(\mathrm{m}^{2}\right)$ & 2.63 \\
\hline Hydraulic diameter (m) & $4.8 \times 10^{-1}$ \\
\hline \multicolumn{2}{|l|}{ Core } \\
\hline Height (m) & 3.6 \\
\hline Hydraulic diameter (m) & $1.22 \times 10^{-2}$ \\
\hline Bypass area $\left(\mathrm{m}^{2}\right)$ & $1.54 \times 10^{-2}$ \\
\hline \multicolumn{2}{|l|}{ Hot-leg } \\
\hline Inner diameter, D (m) & $7.35 \times 10^{-1}$ \\
\hline Length, L (m) & 7.28 \\
\hline $\mathrm{L} / \sqrt{\mathrm{D}}\left(\mathrm{m}^{0.5}\right)$ & 8.48 \\
\hline \multicolumn{2}{|l|}{ U-tube in one $S G$} \\
\hline$\overline{\text { Number }}$ & 5626 \\
\hline Average length (m) & 16.85 \\
\hline Inner diameter (mm) & 15.4 \\
\hline Volume $\left(\mathrm{m}^{3}\right)$ & 18.44 \\
\hline \multicolumn{2}{|l|}{ Pressurizer } \\
\hline Volume $\left(\mathrm{m}^{3}\right)$ & 39.64 \\
\hline Surge-line flow area $\left(\mathrm{m}^{2}\right)$ & $6.38 \times 10^{-2}$ \\
\hline
\end{tabular}

Table 3 Major sequence of Mannshan MSLB

\begin{tabular}{lc}
\hline Events & Second \\
\hline Steady operation & 0 \\
Break occurred & 1000.01 \\
Low pressure scram signal (12.8MPa) & 1000.03 \\
Power tripped & 1000.05 \\
MSIV closed & 1005 \\
ACC injection & 1078 \\
\hline
\end{tabular}

\section{Results}

In this chapter, working flow was assumed isothermal flow during steady state simulation; flow path was the focus in results. As shown in Fig. 3, it was velocity profile at axial section of vessel model in normal operation. When working flow injected into vessel from cold-legs, the flow hit inside wall of vessel (point A), it was affected by flow area and flow direction, most of the flow went down to downcomer, rest of flow went up to top wall of vessel (point B) and made reverse flow. At point C, the flow was slower than point D due to neutron shield panels. In lower plenum, 
flow direction and model shape were major effects on working flow, most of water flowed to bottom along lower plenum wall, it crossed through tie-plates, pipes and core support, finally reached core bottom plate, a few water made turbulent flow and vortex at point E, there was slower flow near the tie-plates. At point F, flow hit core bottom plate and caused vortex. Top profile in Fig. 14 was velocity distribution at horizontal section of downcomer and cold-legs; it located at point A of Fig. 3. It showed that flow spread to both sides along radian of inside wall, some flow hit wall of hot-legs and caused reverse flow (point G). Fig. 3 shows a clear stream line distribution in vessel model, stream lines spread along inside wall and most of them downed to downcomer, the rest struck top wall, then turned down along outside wall. As shown in Fig. 4, there had obvious velocity gradient at two locations between wall and lower tie-plate, which was made by turbulent flow.

As the MSLB event occurred, the vessel temperature was reduced in short time. Fig. 5 shows radial temperature distribution of different height at 1s, 78s and 100s, respectively. The locations of different height in Fig. 5 had a larger variation of flow area or complex geometry, and the non-uniform mixing phenomenon was displayed in the region we interested. Moreover, the locations of different height had its corresponding point at Fig. 3. As shown in Fig. 5, an apparent temperature drop at 78s was found near the primary loop\#2 in downcomer $(\mathrm{H}=0 \mathrm{~m}$, point $\mathrm{A})$. The inference of phenomenon was the DEG at secondary loop\#2. The non-uniform temperature distribution was occurred since the poor-mixing effect and limited space ( $\mathrm{H}=-2.15 \mathrm{~m}$, point $\mathrm{C})$, and the maximum temperature deviation in the downcomer was over $24 \mathrm{~K}$ at $100 \mathrm{~s}$. Non-uniform mixing still had no improvement in lower-plenum. Even there had bigger mixing space and vortex, the axial temperature distribution had the same trend along the height direction $(\mathrm{H}=-6.93 \mathrm{~m}$ and $\mathrm{H}=-7.4 \mathrm{~m}$, point $\mathrm{E}$ ). Consequently, the radial temperature distribution forms a significantly non-uniform temperature distribution with maximum deviation $20 \mathrm{~K}$. It could be forecasted that an obviously temperature difference at the core inlet is inevitable.

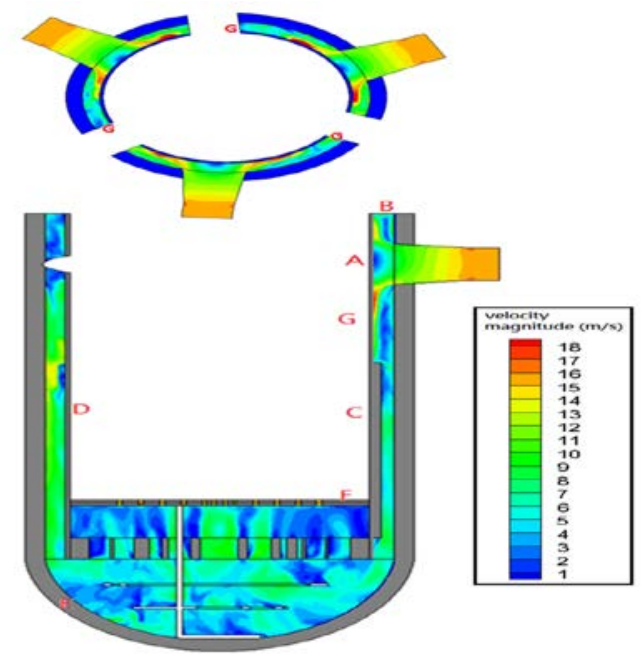

(a) Velocity profile

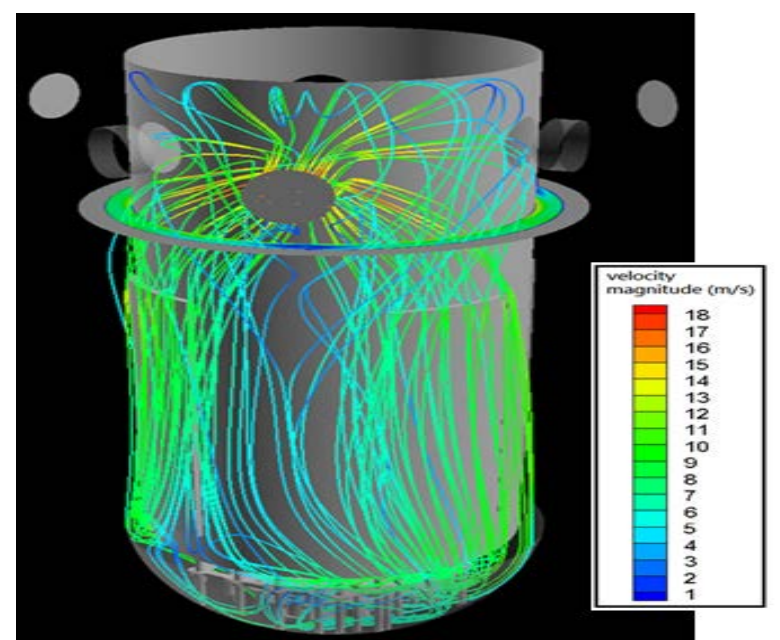

(b) Stream line

Fig. 3. Velocity profile and Stream line results

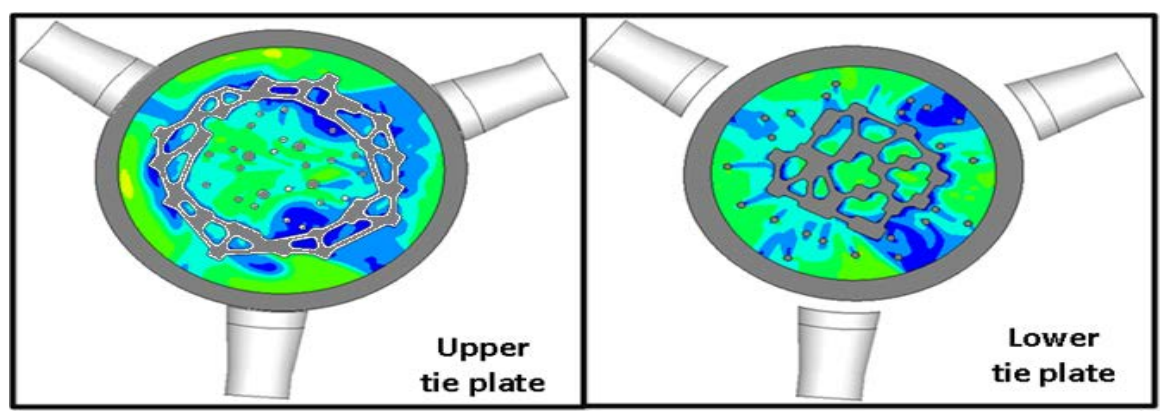

Fig. 4. Velocity profile near the upper and lower tie-plates 


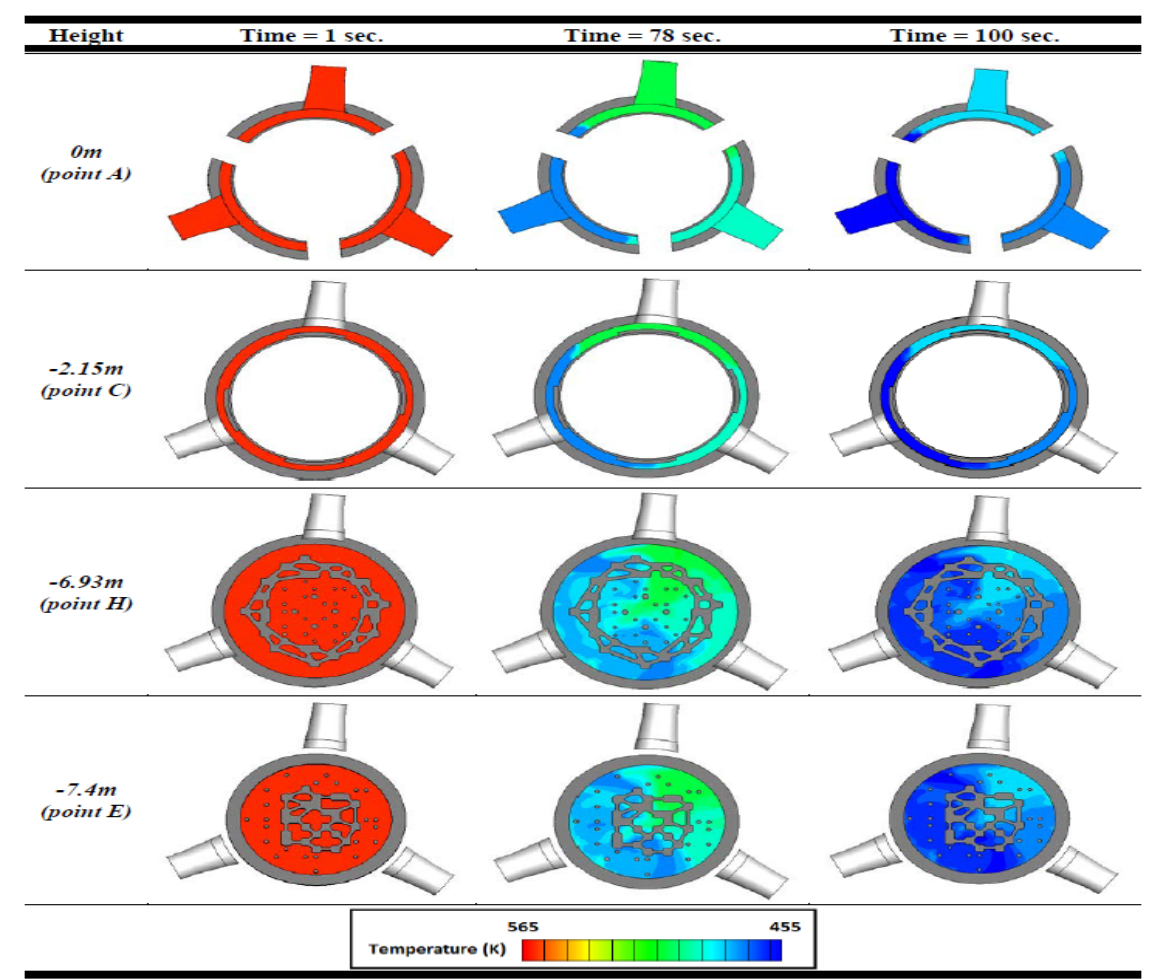

Fig. 5. Radial temperature distribution of different height at 1s, 78s and 100s

\section{Conclusion}

In this study, a three-dimensional partial geometrical TRACE/CFD model of Maanshan NPP RPV has been built to investigate the core flow behaviors and thermal hydraulic phenomena in downcomer and lower-plenum. This model was employed to simulate steady-state conditions and the effects of MSLB transient. Important settings of CFD model were first validated by benchmarking the ROCOM report. In order to provide sufficient transient boundary conditions of MSLB transient for CFD simulations, the Maanshan NPP TRACE model was coupled with the present CFD model. The flow fields inside the core under steady operations were obtained, and several vortexes caused by core geometries were found from the CFD results. In MSLB transient, non-uniform temperature distributions were observed due to the limited space of downcomer and poor mixing of the core flow. The maximum temperature differences under the MSLB transient can reach up to $24 \mathrm{~K}$ and $20 \mathrm{~K}$ in the downcomer and lower-plenum, respectively. This study successfully demonstrated a TRACE/CFD model for Maanshan PWR pressure vessel, and this methodology can be applied to nuclear reactor safety analysis for transient accident events.

\section{References}

[1] U.S. NRC, TRACE V5.840 User’s Manual, 2014.

[2] J.R. Wang, H.T. Lin, Y.H. Cheng, W.C. Wang, C. Shih, TRACE modeling and its verification using Maanshan PWR start-up tests. Annals of Nuclear Energy 36, 527-536, 2009.

[3] ANSYS, FLUENT V12 Theoretical Manual, ANSYS Inc., 2009.

[4] Yu-Ting Ku, Yung-Shin Tseng, Jung-Hua Yang, Shao-Wen Chen, Jong-Rong Wang, Chunkuan Shih, Developments and Applications of TRACE/CFD Model of Maanshan PWR Pressure Vessel, NURETH-16, 2015.

[5] S.V. Patankar, Numerical Heat Transfer and Fluid Flow, MC Graw-Hill, Washington, 1980.

[6] J.P. Van Doormal and G.D. Raithby, Enhancements of the simple method for predicting incompressible fluid flows, Numer. Heat Transf., 1984, 7, 147-163. 